\title{
CIRCLE ACTIONS ON RATIONAL HOMOLOGY MANIFOLDS AND DEFORMATIONS OF RATIONAL HOMOTOPY TYPES
}

\author{
MARTIN RAUSSEN
}

\begin{abstract}
The aim of this paper is to follow up the program set in [LR85, Rau92], i.e., to show the existence of nontrivial group actions ("symmetries") on certain classes of manifolds. More specifically, given a manifold $X$ with submanifold $F$, I would like to construct nontrivial actions of cyclic groups on $X$ with $F$ as fixed point set. Of course, this is not always possible, and a list of necessary conditions for the existence of an action of the circle group $T=S^{1}$ on $X$ with fixed point set $F$ was established in [Rau92]. In this paper, I assume that the rational homotopy types of $F$ and $X$ are related by a deformation in the sense of [All78] between their (Sullivan) models as graded differential algebras (cf. [Sul77, Hal83]). Under certain additional assumptions, it is then possible to construct a rational homotopy description of a $T$-action on the complement $X \backslash F$ that fits together with a given $T$-bundle action on the normal bundle of $F$ in $X$. In a subsequent paper [Rau94], I plan to show how to realize this $T$-action on an actual manifold $Y$ rationally homotopy equivalent to $X$ with fixed point set $F$ and how to "propagate" all but finitely many of the restricted cyclic group actions to $X$ itself.
\end{abstract}

\section{RATIONAL COHOMOLOGY}

Given a (smooth) manifold $X$ and a submanifold $F \subset X$ whose rational homotopy types are related in a sense to be made more precise in several assumptions throughout this paper. In this section, we would like to construct the rational cohomology of a candidate for the orbit space of a $T=S^{1}$-action on the complement $X \backslash F$ such that its (algebraic) boundary fits to the orbit space of a fibrewise $T$-action on the sphere bundle $S \nu$ of the normal bundle $\nu$ of $F$ in $X$.

In order to formulate some assumptions relating $X$ and $F$, we need to describe the rational homotopy types involved as differential graded algebras (dgas) over Q via their minimal models (see, e.g., [BG76, Sul77, GM81, Hal83, AP93]). The following definition is a modification of that in [Ger64] to the category of dgas:

Definition 1.1. Let $(\mathscr{A}, d)$ be a differential graded algebra over $\mathbf{Q}$, and let $e$ denote a (formal) variable in dimension two.

Received by the editors November 4, 1993.

1991 Mathematics Subject Classification. Primary 57S10, 57S17, 55P62.

Key words and phrases. Symmetry, circle group, rational homotopy type, deformation.

Part of the final work with this paper was accomplished while the author was visiting SFB 170 "Geometrie und Analysis" at Göttingen, whose support is gratefully acknowledged. 
(1) The graded algebra $\mathscr{A}[e]=\mathscr{A} \otimes \mathbf{Q}[e]$ together with a differential $d_{[e]}$ is a 1-parameter deformation of $(\mathscr{A}, d)$ if $d_{[e]}$ projects to $d$ under the augmentation map $\varepsilon: \mathscr{A}[e] \rightarrow \mathscr{A}$. More precisely [LR85, Satz 2.8], the differentials $d_{[e]}$ and $d$ are related by a derivation $\tau: \mathscr{A} \rightarrow \mathscr{A}[e]$ such that

- $d_{[e]}(e)=0$

- $d_{[e]}(x)=d(x)+e \cdot \tau(x), x \in \mathscr{A}$

- $d_{[e]} \circ \tau+\tau \circ d=0$.

(2) The trivial deformation of $\mathscr{A}$ is characterized by $\tau=0$.

(3) A 1-parameter deformation of a dga morphism $j:(\mathscr{A}, d) \rightarrow\left(\mathscr{B}, d^{\prime}\right)$ is a dga morphism $j_{[e]}:\left(\mathscr{A}[e], d_{[e]}\right) \rightarrow\left(\mathscr{B}[e], d_{[e]}^{\prime}\right)$ which makes the following diagram commute:

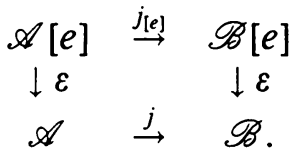

Note that a 1-parameter deformation of $\mathscr{A}$ induces an "algebraic Gysin sequence" [LR85]

$$
\cdots \rightarrow H^{*+1}(\mathscr{A}) \stackrel{t=\tau^{*}}{\longrightarrow} H^{*}(\mathscr{A}[e]) \stackrel{\cdot e}{\longrightarrow} H^{*+2}\left(\mathscr{A}[e] \stackrel{p=\varepsilon^{*}}{\longrightarrow} H^{*+2}(\mathscr{A}) \rightarrow \cdots\right.
$$

as the cohomology long exact sequence of the short exact sequence

$$
0 \rightarrow \mathscr{A}[e] \stackrel{\cdot e}{\longrightarrow} \mathscr{A}[e] \stackrel{\varepsilon}{\rightarrow} \mathscr{A} \rightarrow 0 .
$$

Similarly, a 1-parameter deformation of a dga-morphism induces graded algebra morphisms which fit into a ladder between the corresponding Gysin sequences.

C. Allday defined in [All78] the category of Z/2-graded augmented (KoszulSullivan)-differential algebras (KS2DGA). Let $R=\mathbf{Q}[e]$, and $K=\mathbf{Q}(e)$. In our context, the most important example of such a $\mathbf{Z} / 2$-graded object is $\left(\mathscr{A}(e), d_{(e)}\right)=\left(\mathscr{A}[e] \otimes_{R} K, d_{[e]} \otimes_{R} K\right)$, which, as a graded algebra, is equal to $\mathscr{A} \otimes K$.

Definition 1.2. A dga morphism $j_{[e]}:\left(\mathscr{A}[e], d_{[e]}\right) \rightarrow\left(\mathscr{B}[e], d_{[e]}^{\prime}\right)$ is called a local isomorphism if and only if

$$
j_{[e]} \otimes_{R} \operatorname{id}_{K}:\left(\mathscr{A}(e), d_{(e)}\right) \rightarrow\left(\mathscr{B}(e), d_{(e)}^{\prime}\right)
$$

is a weak homotopy equivalence of $K S 2 D G A$ s, i.e., if it induces an isomorphism in homology.

In that case, the latter morphism is in fact a homotopy equivalence in the category of KS2DGAs [All78, Proposition 2.3]

From now on, we assume that $X$ is a smooth closed simply-connected $n$ dimensional manifold and that $F=\coprod_{i} F_{i} \subset X$ consists of finitely many smooth closed (simply-connected) submanifolds $F_{i}$ of dimension $n_{i}<n$. Their rational minimal models in the sense of [Sul77, GM81, Hal83] are denoted by $(\mathscr{M}(X), d)$, resp. $\left(\mathscr{M}(F), d^{\prime}\right)$. Inclusion induces a dga map $j:(\mathscr{M}(X), d) \rightarrow$ $\left(\mathscr{M}(F), d^{\prime}\right)$. Note that the minimal model of the space $F_{T}=F \times B T$ is given by $\left(\mathscr{M}(F)[e], d^{\prime}\right)$ with trivially extended differential. Furthermore we impose 
Assumption A. The normal bundle $\nu=\nu(F, X)=\amalg \nu\left(F_{i}, X\right)$ of $F$ in $X$ supports a complex structure.

Assumption B. There is a 1-parameter deformation $\left(\mathscr{M}(X)[e], d_{[e]}\right)$ of the minimal model of $X$ and a 1-parameter deformation $j_{[e]}:\left(\mathscr{M}(X)[e], d_{[e]}\right) \rightarrow$ $\left(\mathscr{M}(F)[e], d^{\prime}\right)$ of the inclusion map $j$ into the trivial deformation of $\mathscr{M}(F)$, which is a local isomorphism.

Remark 1.3. In the assumption above, we talk about a specific manifold $F$. Instead, one might just require a deformation into the minimal model of a space $F$ that has a lower cohomological dimension than $X$ itself. It can then be shown along the lines of [Rau92] and the references there, that every component $F_{i}$ is a rational Poincaré complex and that the Poincaré forms of $X$ and $F$ are related. Remark that the proofs in [Rau92] only use the Borel localization theorem, i.e., a situation that is guaranteed by Assumption B. In [Rau94], we shall discuss how to realize those rational Poincaré complexes by manifolds.

The map $j_{[e]}$ in Assumption B should be thought of as an algebraic simulation of the inclusion of Borel spaces $F_{T} \hookrightarrow X_{T}$, where $F$ is the fixed point set of a $T$-action on $X$. The dgas and maps inbetween them may be realized by rational spaces and maps [BG76, Hal83], which we denote by $j_{[e]}: F_{[e]}=F_{(0)} \times$ $B T_{(0)} \rightarrow X_{[e]}$. Also the augmentation maps $\varepsilon:\left(\mathscr{M}(X)[e], d_{[e]}\right) \rightarrow(\mathscr{M}(X), d)$ and $\varepsilon:\left(\mathscr{M}(F)[e], d^{\prime}\right) \rightarrow\left(\mathscr{M}(F), d^{\prime}\right)$ may be realized by maps $p_{x}: X_{(0)} \rightarrow X_{[e]}$, resp. $p_{F}: F_{(0)} \rightarrow F_{(0)} \times B T_{(0)}$.

Interpreting $\left(X_{[e]}, F_{[e]}\right)$ as a pair of rational spaces, Assumption B shows in particular, that $H^{*}\left(X_{[e]}, F_{[e]}\right)$ is a $\mathrm{Q}[e]$-torsion module. As in [Rau92], one may prove

Lemma 1.4. Under Assumption B, the map $j_{[e]}^{*}: H^{n}\left(X_{[e]}\right) \rightarrow H^{n}(X)$ is onto in dimension $n$, if $F \neq \varnothing$.

A choice of a complex structure on $\nu$ induces a (semifree) $T \subset \mathbf{C}^{*}$-action. After choice of a Hermitian metric on $\nu$ and conjugation with the associated exponential map, this action induces a semifree action on a tubular neighborhood $F \subset U \subset X$ with fixed point set $F$. In particular, $\partial U$ becomes a free $T$-manifold $T$-diffeomorphic to the sphere bundle $S \nu$ with orbit space $\partial U / T$ diffeomorphic to $\mathbf{C P \nu}$. Our aim is to construct the homology of a (virtual) orbit space for a (free) $T$-action on the manifold $M=X \backslash U \simeq X \backslash F$ with boundary $\mathbf{C P \nu}$. The first step is:

Lemma 1.5.

$$
H^{*}\left(X_{[e]}, F_{[e]}\right)=H^{*}\left(j_{[e]}\right)= \begin{cases}\mathbf{Q}, & *=n-1, \\ 0, & * \geq n .\end{cases}
$$

Proof. We have to chase the diagram of pairs

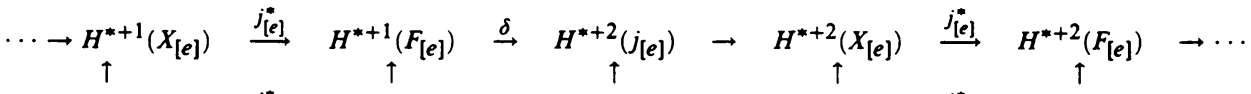

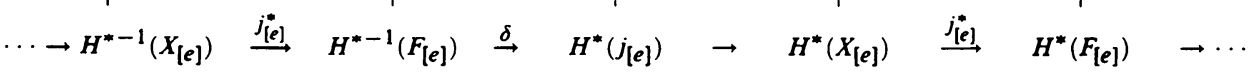

The vertical arrows stand for multiplication by $e$, which is an isomorphism on $H^{*}\left(F_{[e]}\right)$ for all $* \geq \max \left\{n_{i}\right\}-1$ and thus for $* \geq n-3$, and on $H^{*}\left(X_{[e]}\right)$ for $* \geq n-1$. The five lemma shows that it is an isomorphism on $H^{*}\left(j_{[e]}\right)$ for 
$* \geq n$. Furthermore, Assumption B shows that the map $j_{[e]}^{*}$ is an isomorphism for large $*$ and hence for $* \geq n$, which allows to conclude that $H^{*}\left(j_{[e]}\right)=0$ for $* \geq n$.

It follows from Lemma 1.4, that $j_{\text {[e] }}^{n-1}$ is an isomorphism, too, and hence we have $H^{n-1}\left(j_{[e]}\right) \cong \operatorname{coker}\left(j_{[e]}^{n-2}\right)$. On the other hand, by Lemma 1.4, multiplication with $e$ on $H^{n-2}\left(X_{[e]}\right)$ has a 1-dimensional cokernel, generated by an element $x_{[e]}$ with $p_{X}^{*}\left(x_{[e]}\right)=[X] \in H^{n}(X)$, the fundamental class. Hence, $H^{n-1}\left(j_{[e]}\right)$ is a 1-dimensional $Q$-vector space generated by $\delta\left(\frac{1}{e} \cdot j_{[e]}^{*}\left(x_{[e]}\right)\right)$.

The cup-product defines Q-bilinear pairings

$$
I: H^{*}\left(X_{[e]}\right) \otimes H^{n-1-*}\left(X_{[e]}, F_{[e]}\right) \rightarrow H^{n-1}\left(X_{[e]}, F_{[e]}\right) \cong \mathbf{Q}, \quad 0 \leq * \leq n-1,
$$

which in turn yields vector space homomorphisms

$$
i_{[e]}: H^{*}\left(X_{[e]}\right) \rightarrow H_{n-1-*}\left(X_{[e]}, F_{[e]}\right)=\operatorname{Hom}\left(H^{n-1-*}\left(X_{[e]}, F_{[e]}\right) ; \mathbf{Q}\right) .
$$

From now on, we impose:

Assumption C. The inclusion $j$ of $F$ into $X$ induces the trivial map in rational cohomology in positive degrees.

Proposition 1.6. Under Assumption $\mathrm{C}$, the maps $i_{[e]}$ above are onto.

Proof. We use the following notation introduced in [Rau92] (in the absolute case): Let

$$
R(X, F)=p^{*} H^{*}\left(X_{[e]}, F_{[e]}\right) \subset H^{*}(X, F),
$$

and

$$
I(X)=p^{*}\left(\operatorname{Tor}\left(H^{*}\left(X_{[e]}\right)\right)\right)
$$

denote the image of the $\mathrm{Q}[e]$-torsion submodule of $H^{*}\left(X_{[e]}\right)$. First, we show that the map $k^{*}: H^{*}(X, F) \rightarrow H^{*}(X)$ induces an isomorphism

$$
K: H^{*}(X, F) / R(X, F) \rightarrow H^{*}(X) / I(X),
$$

using the commutative diagram

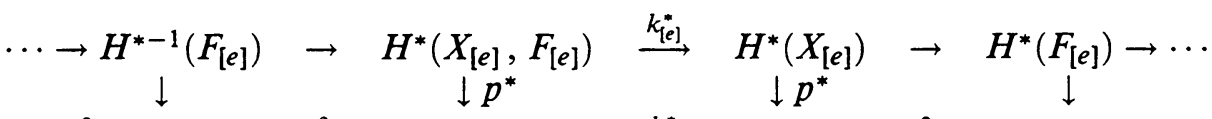

$$
\begin{aligned}
& \cdots \stackrel{0}{\rightarrow} H^{*-1}(F) \quad \stackrel{\delta}{\rightarrow} \quad H^{*}(X, F) \quad \stackrel{k^{*}}{\rightarrow} \quad H^{*}(X) \quad \stackrel{0}{\rightarrow} \quad H^{*}(F) \rightarrow \cdots
\end{aligned}
$$

The quotient map $K$ is well-defined, since, by Assumption B, $H^{*}\left(X_{[e]}, F_{[e]}\right)$ is a $\mathrm{Q}[e]$-torsion module. It is one-to-one, since every torsion element in $H^{*}\left(X_{[e]}\right)$ comes from $H^{*}\left(X_{[e]}, F_{[e]}\right)$, and since $\delta\left(H^{*-1}(F)\right)=\delta(R(F)) \subset$ $R(X, F)$. Finally, $K$ is onto because of Assumption C.

It is shown in [Rau92] that $R(X)=I(X)^{\perp}$ under the Poincare duality form on $H^{*}(X)$. Hence, the latter factors over a nondegenrate pairing $P$ : $H^{*}(X) / I(X) \rightarrow R(X)^{*}$, the Q-vector space dual to $R(X)$. Furthermore, the transgression map $\tau: H^{*}(X, F) \rightarrow H^{*-1}\left(X_{[e]}, F_{[e]}\right)$ induces an isomorphism $\tau: H^{*}(X, F) / R(X, F) \rightarrow \operatorname{ker}(\cdot e) \subset H^{*-1}\left(X_{[e]}, F_{[e]}\right)$. Moreover, the duality forms $P$ and $I$ are linked together by the transgression $\tau$ as follows:

(1) $\tau: \mathbf{Q} \cong H^{n}(X, F) \rightarrow H^{n-1}\left(X_{[e]}, F_{[e]}\right) \cong \mathbf{Q}$ is an isomorphism.

(2) $\tau\left(y \cup p^{*} x\right)=\tau(y) \cup x, y \in H^{*}(X, F), x \in H^{*}\left(X_{[e]}\right)$ [LR85]. 
In the commutative diagram

$$
\begin{array}{cccccc}
H^{*}(X, F) / R(X, F) & \stackrel{K \cong}{\tau} & H^{*}(X) / I(X) & \stackrel{P \cong}{\leftrightarrows} & R_{n-*}(X) \\
\ln (\cdot e) & \subset & H^{*-1}\left(X_{[e]}, F_{[e]}\right) & \stackrel{\stackrel{i_{[e]}^{*}}{\longrightarrow}}{ } & H_{n-*}\left(X_{[e]}\right),
\end{array}
$$

the composite map $H^{*}(X, F) / R(X, F) \rightarrow H_{*}\left(X_{[e]}\right)$ is monomorphic. Hence the adjoint of $i_{[e]}$ has to be injective on $\tau H *(X, F)=\operatorname{ker}(\cdot e)$. We conclude that $i_{[e]}: H^{*}\left(X_{[e]}\right) \rightarrow(\operatorname{ker}(\cdot e))^{*}$, the dual of $\operatorname{ker}(\cdot e)$, is onto.

To show that $i_{[e]}$ is onto $H_{n-1-*}\left(X_{[e]}, F_{[e]}\right)$, pick an element

$$
z \in \operatorname{ker}\left(\cdot e^{i}\right) \backslash \operatorname{ker}\left(\cdot e^{i-1}\right) \subset H^{*}(X, F) .
$$

Since $e^{i-1} \cdot z \in \operatorname{ker}(\cdot e)$, there is an element $x \in H^{*}\left(X_{T}\right)$ such that $z \cup e^{i-1}$. $p^{*} x=e^{i-1} \cdot z \cup p^{*} x \neq 0 \in H^{n-1}\left(X_{[e]}, F_{[e]}\right)$.

For $M=X \backslash F$, Alexander duality suggest the following

Definition 1.7. $H_{[e]}^{*}(M)=H_{n-1-*}\left(X_{[e]}, F_{[e]}\right)$.

In particular, $H_{[e]}^{*}(M)=0$ for $*<0$ and $* \geq n$. The surjections $i_{[e]}$ : $H^{*}\left(X_{[e]}\right) \rightarrow H_{[e]}^{*}(M)$ can be used to give the latter graded Q-vector space the structure of a graded ring with a map $(\cdot e)^{*}$ of degree 2 as a graded $\mathbf{Q}[e]$-quotient algebra of $H^{*}\left(X_{[e]}\right)$.

Our next goal is to define connecting homomorphisms in a Gysin type long exact sequence

$$
\cdots H^{*-1}(M) \stackrel{t_{M}}{\longrightarrow} H_{[e]}^{*-2}(M) \stackrel{\cdot e}{\rightarrow} H_{[e]}^{*}(M) \stackrel{p_{M}}{\longrightarrow} H^{*}(M) \cdots .
$$

This is quite easy using Alexander duality $A: H^{*}(X \backslash F) \cong H^{*}(X \backslash D \nu) \cong$ $H_{n-*}(X, D \nu) \cong H_{n-*}(X, F)$. In detail, $t_{M}$, resp. $p_{M}$, are given by the compositions

$$
\begin{gathered}
t_{M}: H^{*-1}(X \backslash F) \stackrel{A}{\longrightarrow} H_{n-*+1}(X, F) \stackrel{p_{*}}{\longrightarrow} H_{n-*+1}\left(X_{[e]}, F_{[e]}\right) \stackrel{\cong}{\longrightarrow} H_{[e]}^{*-2}(X \backslash F), \\
p_{M}: H_{[e]}^{*}(X \backslash F) \stackrel{\cong}{\longrightarrow} H_{n-*-1}\left(X_{[e]}, F_{[e]}\right) \stackrel{t^{*}}{\longrightarrow} H_{n-*}(X, F) \stackrel{A^{-1}}{\longrightarrow} H^{*}(X \backslash F) .
\end{gathered}
$$

As in [LR85, Lemma 2.1.c], one may show inductively

Lemma 1.8. The Gysin type sequence (1.2) is exact.

Next, we have to simulate the inclusion of the boundary $C P \nu \subset(X \backslash F) / T$ in case of a $T$-action by an algebraic counterpart. Geometry imposes an additional requirement: Let $F=\coprod_{i} F_{i}, \mathbf{C P \nu}=\coprod_{i} \mathbf{C} P \nu_{i}$ denote decomposition into connected components. Then $H^{n-2}(\mathbf{C} P \nu) \cong \bigoplus_{i} H^{n-2}\left(\mathbf{C} P \nu_{i}\right) \cong \bigoplus_{i} \mathbf{Q}$ by evaluation at (properly chosen) fundamental classes. Adding over the components yields a map $E: H^{n-2}(\mathbf{C P \nu}) \rightarrow \bigoplus_{i} \mathbf{Q} \rightarrow \mathbf{Q}$. Let $p_{[e]}: \mathbf{C} P \nu \rightarrow F_{T}$ denote the Borel construction applied to the inclusion $S \nu \hookrightarrow D \nu$, which is a map over $F$. 
Assumption D. The sequence

$$
0 \rightarrow H^{n-2}\left(X_{[e]}\right) \stackrel{j_{[e]}^{*}}{\longrightarrow} H^{n-2}\left(F_{[e]}\right) \stackrel{E \circ p_{[e]}^{*}}{\longrightarrow} \mathbf{Q} \rightarrow 0
$$

is exact.

Remark 1.9. (1) The proof of Lemma 1.5 shows, that the cokernel of $j_{[e]}^{*}$ is isomorphic to $\mathbf{Q}$ under Assumption B. Hence, the assumption only specifies the image of $j_{[e]}^{*}$ in dimension $n-2$.

(2) If there is a semifree $T$-action on $X$ with fixed point set $F$ and with $M \simeq X \backslash F$, then the sequence in Assumption $\mathrm{D}$ is in fact exact, since

(a) $H^{n-2}\left(X_{[e]}, F_{[e]}\right) \cong H^{n-2}(M / T, \mathbf{C P} \nu) \stackrel{\cdot e}{\cong} H^{n}(M / T, \mathbf{C P} \nu)=0$ (use excision and $H^{n-1}(M, S \nu) \cong H_{1}(M)=0$ ).

(b) The following diagram commutes:

$$
\begin{array}{rlll}
H^{n-2}\left(X_{[e]}\right) \stackrel{j_{[e]}^{*}}{\longrightarrow} & \begin{array}{c}
H^{n-2}\left(F_{[e]}\right) \\
\downarrow p_{[e]}^{*}
\end{array} & \stackrel{\delta}{\longrightarrow} & H^{n-1}\left(X_{[e]}, \underset{\downarrow l e]}{F}\right) \cong \mathbf{Q} \rightarrow 0 \\
& H^{n-2}(\mathbf{C P \nu}) & \stackrel{\delta=E}{\longrightarrow} & H^{n-1}(M / T, \mathbf{C P} \nu) \cong \mathbf{Q}
\end{array}
$$

(After proper choices of fundamental classes, the bottom map $\delta$ corresponds to the map $E$ above.)

Corollary 1.10. Assumption D implies: $\operatorname{ker}\left(i_{[e]}\right) \subseteq \operatorname{ker}\left(p_{[e]}^{*} \circ j_{[e]}^{*}\right)$, i.e., $p_{[e]}^{*} j_{[e]}^{*} z=$ 0 for all $z \in H^{*}\left(X_{[e]}\right)$ satisfying $z \cup H^{n-*-1}\left(X_{[e]}, F_{[e]}\right)=0 \in H^{n-1}\left(X_{[e]}, F_{[e]}\right)$. Hence, there is a well-defined ring homomorphism $k_{[e]}$ completing the square

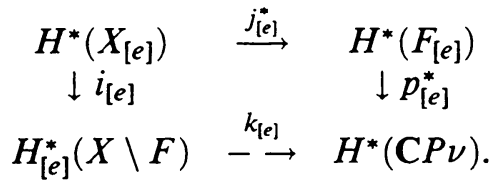

Remark 1.11. If the fixed point set $F$ is connected, the conclusion above has the following geometric interpretation: $j_{[e]}^{*}(z) \in H^{*}\left(F_{[e]}\right)$ is a multiple of the total Chern class

$$
C(\nu)=e^{\frac{n-m}{2}}+c_{1}(\nu F) \cdot e^{\frac{n-m}{2}-1}+c_{2}(\nu F) \cdot e^{\frac{n-m}{2}-2}+\cdots+c_{\frac{n-m}{2}}(\nu F) .
$$

Proof. The existence of $k_{[e]}$ above is equivalent to the first statement in the corollary. The latter is trivially true for $* \geq n-1$, since $H^{*}(\mathbf{C P \nu})=0$.

Now, suppose $z \in H^{*}\left(X_{[e]}\right), * \leq n-2$, and $z \cup H^{n-*-1}\left(X_{[e]}, F_{[e]}\right)=0$. Let $\delta$ denote the connecting homomorphism $\delta: H^{*}\left(F_{[e]}\right) \rightarrow H^{*+1}\left(X_{[e]}, F_{[e]}\right)$. For all $s \in H^{n-*-2}\left(F_{[e]}\right)$, the condition above implies: $\delta\left(j_{[e]}^{*}(z) \cup s\right)=z \cup \delta s=0$. Hence, for every such $s$, there is an element $u \in H^{n-2}\left(X_{[e]}\right)$ with $j_{[e]}^{*}(u)=$ $j_{[e]}^{*}(z) \cup s$.

Thus, for every $\sigma_{i} \in H^{n-*-2}\left(\mathbf{C} P \nu_{i}\right)$, Assumption D implies:

$$
E\left(p_{[e]}^{*}\left(j_{[e]}^{*}(z)\right) \cup \sigma_{i}\right)=0 .
$$

Since $p_{[e]}^{*}\left(j_{[e]}^{*}(z)\right) \cup \sigma_{i}$ lives in $H^{n-2}\left(\mathbf{C P} \nu_{i}\right)$, it has to be trivial itself. Using Poincaré duality for $\mathrm{CP} \nu_{i}$, we conclude that $p_{[e]}^{*}\left(j_{[e]}^{*}(z)\right)=0$. 
We finish this section with two lemmas that show that our algebra behaves as the cohomology of an orbit space with respect to quotient maps and transgressions:

Lemma 1.12. Let $k: S \nu \hookrightarrow X \backslash F$ denote the inclusion map. The diagram

$$
\begin{array}{ccc}
H_{[e]}^{*}\left(X_{[e]}\right) & \stackrel{k_{[e]}}{\longrightarrow} & H^{*}(\mathbf{C P} \nu) \\
\downarrow p_{M}^{*} & & \downarrow p_{\nu}^{*} \\
H^{*}(X \backslash F) & \stackrel{k^{*}}{\rightarrow} & H^{*}(S \nu)
\end{array}
$$

commutes. If $F$ is rationally contractible in $X$, i.e., $j^{*}=0$ for $*>0$, both compositions are trivial.

Proof. The diagram of the lemma embeds into

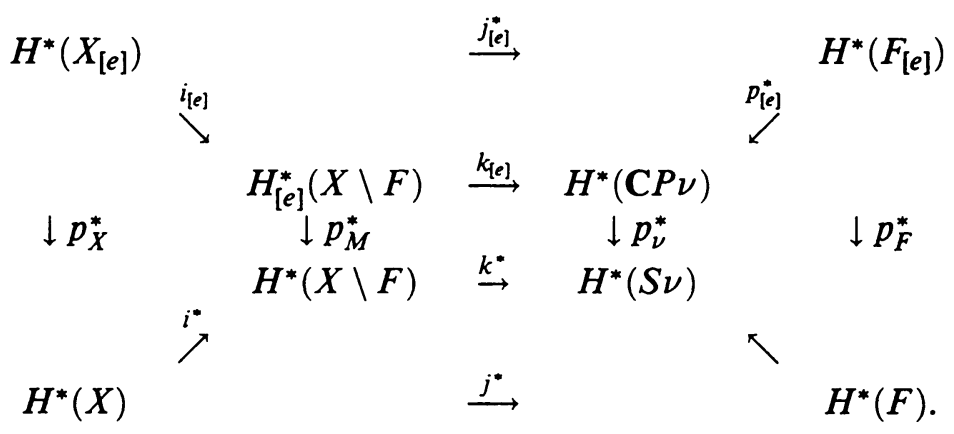

Since $i_{[e]}$ is surjective, it is enough to see that all the outer diagrams commute. This is true by definition apart from the left parallelogram, which rewrites as

$$
\begin{array}{ccc}
H^{*}\left(X_{[e]}\right) & \stackrel{i_{[e]}}{\longrightarrow} & H_{n-*-1}\left(X_{[e]}, F_{[e]}\right) \\
\downarrow p_{X}^{*} & & \downarrow t^{*} \\
H^{*}(X) & \longrightarrow & H_{n-*}(X, F),
\end{array}
$$

where the horizontal arrows denote evaluation at the fundamental classes. From the Gysin sequence (1.2) it is easy to see (as in [LR85]) that $t: H^{n}(X, F) \rightarrow$ $H^{n-1}\left(X_{[e]}, F_{[e]}\right)$ is an isomorphism, and commutativity of the last diagram thus follows from:

$$
t\left(p_{X}^{*}(y) \cup z\right)=y \cup t(z), y \in H^{*}\left(X_{[e]}\right), z \in H^{n-*}(X, F),
$$

see [LR85, p. 552].

Lemma 1.13. The following diagram commutes:

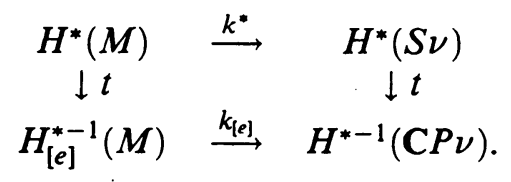


Proof. Embed the diagram of the lemma as the center of the following diagram:

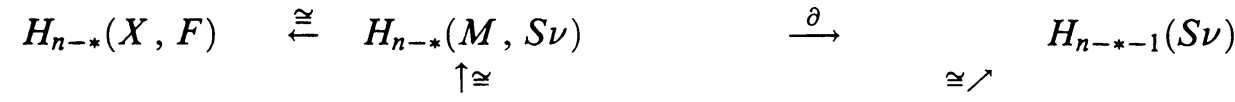

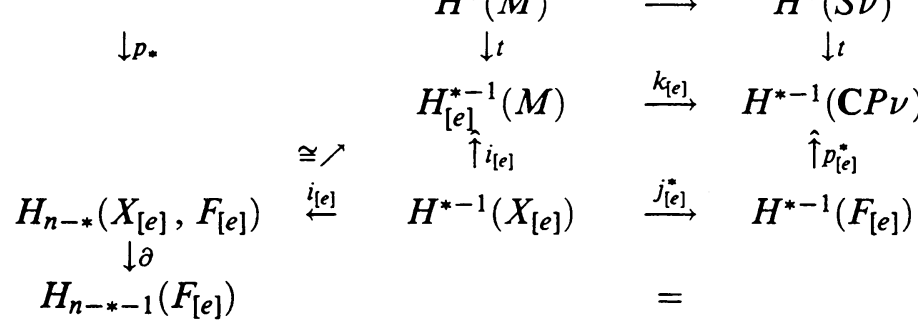

$$
\begin{aligned}
& H^{*}(M) \\
& \downarrow p *
\end{aligned}
$$

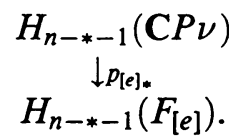

We have to check commutativity of the outer and of the lower "rectangles"; commutativity of the smaller interior diagrams is by definition or routine.

The outer diagram commutes, since it is dual to part of the following commutative diagram:

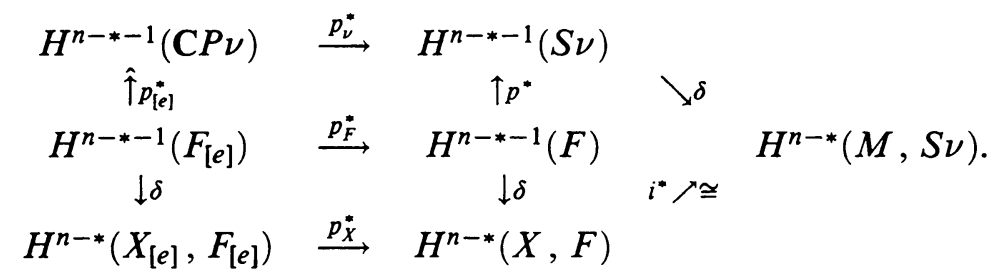

Now to the lower part of the diagram: Let $y \in H^{*-1}\left(X_{[e]}\right)$. Moving to the left, it corresponds to the linear form $\left(z \mapsto y \cup \delta z \in H^{n-1}\left(X_{[e]}, F_{[e]}\right) \cong \mathbf{Q}\right)$ on $H^{n-*-1}\left(F_{[e]}\right)$. Under the path to the right it corresponds to the map $(z \mapsto$ $\left.E\left(p_{[e]}^{*} j_{[e]}^{*} y \cup p^{*} z\right) \in \mathbf{Q}\right)$. Both come from the map $\left(z \mapsto j_{[e]}^{*} y \cup z \in H^{n-2}\left(F_{[e]}\right)\right)$ by composition with $\delta$, resp. $p_{[e]}^{*}$. According to Assumption $\mathrm{D}$, the maps $\delta$ and $E \circ p_{[e]}^{*}: H^{n-2}(\mathbf{C} P \nu) \rightarrow \mathbf{Q}$ agree upto a nontrivial rational factor, which can be eliminated by a change of the fundamental class $H^{n-1}\left(X_{[e]}, F_{[e]}\right)$.

\section{RATIONAL hOMOtOPY: PERTURBING SPACES AND MAPS}

It was the plan of the preceding section to describe the cohomology of a potential orbit space of a $T$-action on $M=X \backslash F$ as a perturbation (quotient) of the cohomology of the potential Borel space $X_{[e]}$. Similarly, the cohomology of the inclusion map from $\mathbf{C P \nu}$ into it was obtained by a perturbation (quotient) of the deformation map $j_{[e]}^{*}: H^{*}\left(X_{[e]}\right) \rightarrow H^{*}\left(F_{[e]}\right)$. In this section, we are going to refine this method to rational homotopy [Sul77, BG76, GM81, Hal83], which makes additional hypotheses necessary.

First, we show that certain conditions on the (co)-connectivity of $X$, resp. $F$, imply that the rational homotopy of $X$ and $M=X \backslash F$ are closely related. As a consequence, it turns out, that a minimal model of a space $M_{[e]}$ may be constructed as a perturbation of $\mathscr{M}\left(X_{[e]}\right)$, and similarly a rational map $\mathrm{CP \nu} \rightarrow$ $M_{[e]}$.

We begin by presenting some necessary easy (and probably well-known) lemmas from rational homotopy theory. The first question is: Given a map 
$f: A \rightarrow X$. To which extent does the rational homotopy of $X$ together with the rational cohomology of the map $f$ determine the rational homotopy of $A$, or, in other words, is the rational homotopy of $A$ "formal, given that of $X$ "? The reader should have in mind the case $A=X \backslash F$.

Proposition 2.1. Let $f: A \rightarrow X$ be a map between 1-connected $C W$-complexes with

$$
\begin{aligned}
& H^{*}(X ; \mathbf{Q})=0, \quad * \leq i ; \quad H^{*}(f ; \mathbf{Q})=0, \quad * \leq k ; \\
& H^{*}(A ; \mathbf{Q})=0, \quad * \geq j \text {. }
\end{aligned}
$$

For $j \leq \min \{i+k, 2 k-1\}$, the rational homotopy of $A$ is determined by that of $X$ and by the (canonically induced) $i^{*}: H^{*}(f) \rightarrow H^{*}(X)$.

Proof. Let $\mathscr{A}$ denote the functor which to a simplicial complex associates its rational PL de Rham complex [Sul77, Hal83]. Regard the composition $\mathscr{M}(X) \rightarrow \mathscr{A}(X) \rightarrow \mathscr{A}(A)$ of a model map for $X$ and the map induced by $f$. A model for $A$ extending $\mathscr{M}(X)$ can be obtained from the Postnikov tower of the map $f$ turned into a fibration $F_{f} \rightarrow A \rightarrow X$. This has been made explicit in the thesis of Grivel, see [Hal83]. In our case, $F_{f}$ is rationally $(k-1)$-connected, and hence, up to dimension $2 k-2$, the following diagram consists of isomorphisms:

$$
\mathscr{M}\left(F_{f}\right)(2 k-2) \cong \begin{aligned}
\pi^{*}\left(F_{f}\right) \cong s^{-1} \pi^{*}(f) \\
\uparrow h^{*} \cong \\
H^{*}\left(F_{f}\right) \cong s^{-1} h^{*}(f)
\end{aligned} \quad(* \leq 2 k-2) .
$$

where $\pi^{*}=\operatorname{Hom}\left(\pi_{*} ; \mathbf{Q}\right), s$ denotes a degree 1 suspension, and $h^{*}$ denotes the dual of the Hurewicz homomorphism. Let $l: \mathscr{M}\left(F_{f}\right)(2 k-2) \rightarrow s^{-1} H^{*}(f)$ denote the obvious composition. Lift the cohomology of the map

$$
\mathscr{M}\left(F_{f}\right)(2 k-2) \stackrel{l}{\rightarrow} s^{-1} H^{*}(f) \stackrel{i^{*}}{\rightarrow} s^{-1} H^{*}(X)
$$

to get a map $d_{f}: \mathscr{M}\left(F_{f}\right)(2 k-2) \rightarrow \mathscr{M}(X)$ of degree 1 and define

$$
\mathscr{M}^{\prime}(A)=\left(\mathscr{M}(X) \otimes \mathscr{M}\left(F_{f}\right)(2 k-2), d_{X} \otimes d_{f}\right) .
$$

It is then easy to write down a dga map $\mathscr{M}^{\prime}(A) \rightarrow \mathscr{A}(A)$ which is a weak equivalence up to dimension $\min \{i+k, 2 k-1\}$; the first bound is needed to exclude mixed products in cohomology. Since $H^{*}(A ; \mathbf{Q})=0, * \geq j$, the rational homotopy of $A$ can then be determined from $\mathscr{M}^{\prime}(A)$ in a purely formal way [Sul77].

Corollary 2.2. If $f_{*}: \pi_{*}(A) \otimes \mathbf{Q} \rightarrow \pi_{*}(X) \otimes \mathbf{Q}$ is onto for $k<* \leq k^{\prime}$, then $\mathscr{M}^{\prime}(A)$ above is a minimal model through dimension $k^{\prime}-1$. If $f_{*}: H_{*}(A ; \mathbf{Q}) \rightarrow$ $H_{*}(X ; \mathbf{Q})$ is onto for $k<* \leq k^{\prime}$, then the differential $d_{f}$ may be chosen to be trivial through dimension $k^{\prime}-1$. 
Proof. Under the assumptions of the corollary, the lower horizontal maps in the following diagram are trivial:

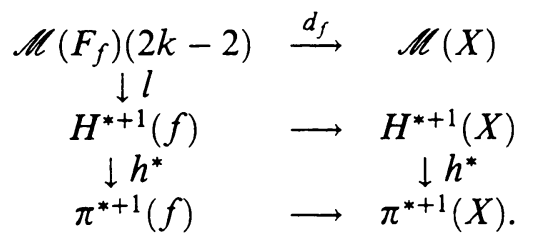

Corollary 2.3. Let $f: A \rightarrow X$ be as in (2.1). If $X$ is formal, so is $A$.

Proof. The diagram

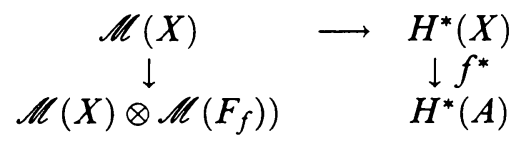

with a rational homotopy equivalence on top can easily be extended to a weak equivalence up to dimension $j-1$ in the bottom line as in (2.1). An extension to dimensions $\geq j$ is formal as well.

Definition 2.4. Let $X$ be an $n$-dimensional connected 1-connected manifold, $F=\amalg F_{j}$ a collection of disjoint 1-connected submanifolds, $\operatorname{dim} F_{j}=m_{j}, m=$ $\max \left\{m_{j}\right\}$. Define $c X=\max \left\{k \mid H^{k}(X ; \mathbf{Q})=0\right\}$ and

$$
c F= \begin{cases}\max \left\{k \mid H^{k}(F ; \mathbf{Q})=0\right\}, & F \text { connected } \\ -1, & \text { else. }\end{cases}
$$

Assumption E. All of the following inequalities are satisfied:

$$
m \leq c X+c F, m<2 \cdot c X-1,2 \cdot m \leq n+2 \cdot c F-1,2 \cdot m \leq n+c X .
$$

Corollary 2.5. Under Assumption E, the rational homotopy type of $M=X \backslash F$ has cohomological dimension $d=n-\min \{c X, c F+1\}$ and is determined by that of $X$ and by the map $i^{*}: H^{*}(X, M) \rightarrow H^{*}(X)$.

Proof. Use the Thom isomorphism (with $t\left(\nu_{i}\right)$ denoting the Thom class of $\nu_{i}$ ), Alexander duality, and excision to obtain:

$$
\begin{aligned}
H^{*}(X, M) & \cong \bigoplus_{i} H^{*}\left(D \nu_{i}, S \nu_{i}\right) \\
& \cong \bigoplus_{i} H^{*-n+m_{i}}\left(F_{i}\right) \cdot t\left(\nu_{i}\right)=0, \quad * \leq n-m-1 ;
\end{aligned}
$$

$$
H^{*}(M) \cong H_{n-*}(M, S \nu) \cong H_{n-*}(X, F)=0, * \geq n-c F-1 \text { and } * \geq n-c X \text {. }
$$

Use (2.1) with $k=n-m-1, j=\min \{n-c F-1, n-c X\}$.

Corollary 2.6. If, in addition to Assumption E, the inclusion of $F$ into $X$ is trivial, i.e., it factors over a point in rational homotopy, then a minimal model of $M=X \backslash F$ is obtained from $\mathscr{M}(X) \otimes \Lambda s^{-1} H^{*}(X, M)$ with trivial differential on the second factor by killing cohomology in dimensions greater than or equal to $n-\min \{c X, c F+1\}$. 
Corollary 2.7. If, in addition to Assumption $\mathrm{E}, \operatorname{dim} X \leq 4 \cdot c X+2$, then both $X$ and $M=X \backslash F$ are formal.

Proof. Combine [Mil79] with (2.3).

The perturbation of $X$ into $M \simeq X \backslash F$ that we have obtained above can be modified on the "Borel space level" to obtain a rational space $M_{[e]}$. We use the cohomological constructions from section 1 as a guide just as in [LR85, cf. in particular Satz 2.9]. We construct a minimal dga $\left(\mathscr{M}\left(M_{[e]}\right), \delta\right)$ such that $\mathscr{M}\left(M_{[e]}\right) \cong \mathscr{M}(M) \otimes \mathscr{M}\left(B T_{(0)}\right) \cong \mathscr{M}(M) \otimes \mathrm{Q}[e]$ as a graded algebra, and such that the differential $\delta$ on $\mathscr{M}\left(M_{[e]}\right)$ satisfies the requirements of Assumption B for a deformation. Furthermore, its cohomolgy is the same as that of the candidate $H_{[e]}^{*}(M)$ constructed in section 1 .

Proposition 2.8. Under Assumptions $\mathrm{C}$ and $\mathrm{E}$, there is a rational space $M_{[e]}$ over $B T_{(0)}$ and a rational homotopy equivalence from $M$ into the pullback of the diagram

$$
\begin{aligned}
& *=E T_{(0)} \\
& \downarrow \\
& M_{[e]} \rightarrow \quad B T_{(0)} .
\end{aligned}
$$

Furthermore, there is an isomorphism $\psi: H^{*}\left(M_{[e]}\right) \rightarrow H_{[e]}^{*}(M)$ such that the following diagram commutes:

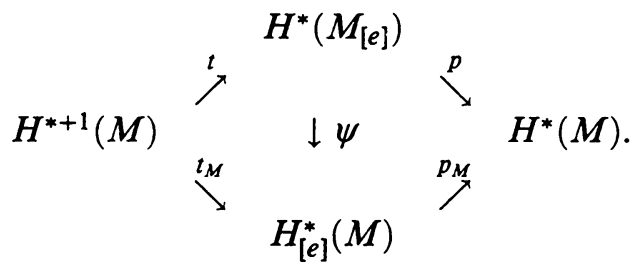

Proof. We want to obtain a diagram

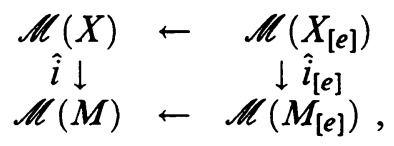

where $\mathscr{M}\left(M_{[e]}\right) \cong \mathscr{M}(M) \otimes \mathbf{Q}[e]$ as a graded algebra, and $\mathscr{M}(M) \cong(\mathscr{M}(X) \otimes$ $\left.\Lambda s^{-1} H^{*}(X, M), d_{X} \otimes 0\right)$ as a dga, up to the cohomological dimension $d$ from Corollary 2.5. (From Corollary 2.6 we know that the differential on the second factor is trivial.)

We have to find a perturbed differential on $\mathscr{M}\left(M_{[e]}\right):$ First, we define a new differential $\delta_{2}=e \cdot \tau: s^{-1} H^{*}(X, M) \rightarrow \mathscr{M}\left(X_{[e]}\right)$ such that the map $\tau^{*}$ in cohomology makes the following diagram commute:

$$
\begin{array}{ccc}
s^{-1} H^{*}(X, M) & \hookrightarrow & H^{*}(M) \\
\tau^{*} \downarrow & & \downarrow t_{M} \\
H^{*+1}\left(X_{[e]}\right) & \vec{i}_{[e]} & H_{[e]}^{*+1}(M) .
\end{array}
$$

Extend $\tau$ to $\Lambda s^{-1} H^{*}(X, M)$ as a derivation, and define $\delta=d_{X} \otimes \delta_{2}$ to be the new differential on $\left.\mathscr{M}\left(M_{[e]}\right)\right)$ up to dimension $d$. Above the cohomological dimension $d$, the derivation can be extended formally-killing 
cohomology -in the same way as in the proof of [LR85, Satz 2.9]. The resulting cohomology $H^{*}\left(M_{[e]}\right)=H^{*}\left(\mathscr{M}\left(M_{[e]}\right)\right.$ is a quotient of $H^{*}\left(X_{[e]}\right)$ which is isomorphic to $H_{[e]}^{*}(M)$, since the new differential annihilates precisely the kernel of $i_{[e]}: H^{*}\left(X_{[e]}\right) \rightarrow H_{[e]}^{*}(M)$. The map $i_{[e]}$ factors over $H^{*}\left(M_{[e]}\right)$ to yield the map $\psi$ above.

Remark 2.9. (1) The proof is by induction on degrees and works only for minimal algebras with decomposable differentials. I do not know whether (2.8) is true without Assumption C.

(2) One can show as in [LR85], that the construction above ends up with a formal space $X_{[e]}$ when feeded with a formal space $X$.

Note for the sake of completeness:

Lemma 2.10. Let $\nu \downarrow F$ denote a vector bundle over the space $F$ with minimal model $\mathscr{M}(F)$.

(1) If $\nu^{l}$ is a real vector bundle with Euler class $e(\nu) \in H^{k}(F)$, a minimal model for the sphere bundle $S(\nu)$ is given by

$$
\mathscr{M}(S \nu)=\left(\mathscr{M}(F) \otimes \bigwedge(s), d \otimes d_{1}\right),
$$

where $|s|=l-1$ and $d_{1}(s)$ is a cocyle representing $e(\nu)$ in cohomology.

(2) If $\nu^{k}$ is a complex vector bundle with total Chern class $C(\nu) \in H^{* *}(F)[e]$ (cf. 1.11), a minimal model of the projective bundle $\mathrm{CP \nu}$ is given by

$$
\mathscr{M}(\mathbf{C P \nu})=\left(\mathscr{M}(F) \otimes \bigwedge(e, s), d \otimes d_{2}\right),
$$

where $|e|=2,|s|=2 k-1, d_{2}(e)=0$, and $d_{2}(s)$ is a cocycle representing $C(\nu)$.

We finish this section by lifting the map $k_{[e]}: H_{[e]}^{*}(M) \rightarrow H^{*}(\mathbf{C P \nu})$ from Corollary 1.10 to rational homotopy.

Proposition 2.11. Under Assumptions C-E, there is a dga map $K_{[e]}: \mathscr{M}\left(M_{[e]}\right) \rightarrow$ $\mathscr{M}(\mathbf{C P \nu})$ inducing $k_{[e]}$ in cohomology and fitting into the diagram

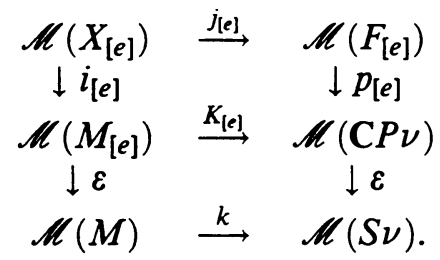

Proof. Since $i_{[e]}^{*}$ is supposed to be onto, we only have to extend the map $p_{[e]} \circ j_{[e]}: \mathscr{M}\left(X_{[e]}\right) \rightarrow \mathscr{M}(\mathbf{C P \nu})$ to $s^{-1} H^{*}(f)$ to get a dga map defined on $\mathscr{M}\left(M_{[e]}\right)$, i.e., it has to be defined on the generators of $s^{-1} H^{*}(f)(d)$ in a way that commutes with differentials. Above the cohomological dimension, it can always be extended to a dga map.

Let $y$ denote a generator from $s^{-1} H^{*}(X, M)$, representing a cocycle in $\mathscr{M}(M)$. We want to define $K_{[e]}(y)=k(y)+e \cdot k^{\prime}(y)$, where $k^{\prime}$ has to be defined as a map of degree 2 in such a way that $K_{[e]}$ commutes with differentials. Note, that the lower diagram of the proposition commutes by definition. It turns out that $K_{[e]}$ is a dga map if and only if $d_{[e]} k^{\prime}(y)=K_{[e]}(\tau y)-\tau k(y)$, where $d_{[e]}$ 
denotes the differential on $\mathscr{M}(\mathbf{C P \nu})$ and $\tau$ denotes the transgressions in that algebra, resp. in $\mathscr{M}\left(M_{[e]}\right)$. The element on the right side is in fact a coboundary by (1.13).

\section{EXCISION AND DUALITY}

For the future development in [Rau94], it is important to ensure that the rational homotopy type of the pair given by the map $K_{[e]}$ of Proposition 2.11 from $C P \nu$ into $M$ behaves like a manifold with boundary, i.e., satifies Alexander duality. Furthermore, it is preferable to have excision properties at hand as in the situation of an actual $T$-action. In this section we assume the situation of Propositions 2.8 and 2.11, in particular, there is a commutative diagram of (rational) spaces:

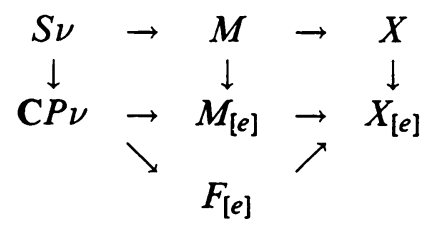

Lemma 3.1. The bottom maps give rise to an isomorphism $H^{*}\left(X_{[e]}, F_{[e]}\right) \rightarrow$ $H^{*}\left(M_{[e]}, \mathbf{C P \nu}\right)$. In particular,

$$
H^{*}\left(M_{[e]}, \mathbf{C P \nu}\right) \cong \begin{cases}0, & * \geq n, \\ \mathbf{Q}, & *=n-1 .\end{cases}
$$

Proof. By Proposition 2.11, there is an algebraic Gysin sequence

$$
\cdots H^{*}(M, S \nu) \rightarrow H^{*-1}\left(M_{[e]}, \mathrm{C} P \nu\right) \rightarrow H^{*+1}\left(M_{[e]}, \mathrm{C} P \nu\right) \rightarrow \cdots
$$

which is connected to the Gysin sequence of $(X, F)$ and $\left(X_{[e]}, F_{[e]}\right)$ by a ladder of homomorphisms. Excision yields an isomorphism $H^{*}(X, F) \cong H^{*}(M, S \nu)$ at every third term. As in [LR85], p. 564, the lemma follows by induction.

Lemma 3.2. Evaluation at a fundamental class in $H^{*}\left(M_{[e]}, \mathbf{C P \nu}\right)$ yields an isomorphism

$$
H^{*}\left(M_{[e]}\right) \rightarrow H_{n-1-*}\left(M_{[e]}, \mathrm{CP \nu}\right) \cong H_{n-1-*}\left(X_{[e]}, F_{[e]}\right) .
$$

Proof. Algebraic Gysin sequence, Alexander duality on total spaces, and induction as in [LR85], p.568.

Lemma 3.2 is in fact the justification for choosing $H_{n-1-*}\left(X_{[e]}, F_{[e]}\right)$ as the "cohomology candidate" $H_{[e]}^{*}(M)$.

Lemma 3.3. The map $H^{*}\left(X_{[e]}, M_{[e]}\right) \rightarrow H^{*}\left(F_{[e]}, \mathrm{CP \nu}\right)$ is an isomorphism.

Proof. Form the obvious ladder between the two Gysin sequences, use excision $H^{*}(X, M) \cong H^{*}(F, S \nu)$ on every third term, and induction.

As in [Rau92], Proposition 3.1.3, one can conclude easily that the long exact sequence of the pair $\left(X_{[e]}, M_{[e]}\right)$ splits into short exact pieces

$$
0 \rightarrow H^{*}\left(X_{[e]}, M_{[e]}\right) \rightarrow H^{*}\left(X_{[e]}\right) \rightarrow H^{*}\left(M_{[e]}\right) \rightarrow 0 .
$$




\section{EXAMPLE: ACtIONS ON HIGHLY-CONNECTED MANIFOLDS WITH ISOLATED FIXED POINTS}

In [LR85], we showed the existence of $T_{(0)}$-actions on every CW-complex with the rational homotopy type of a sufficiently connected manifold with vanishing Euler-characteristic and index (and satisfying an additional technical assumption concerning Pontryagin classes). In this section, we want to generalize these results to manifolds with nonnegative Euler-characteristic and vanishing index, cf. Proposition 4.5. In [Rau94], they will be applied to the construction of semifree actions of cyclic groups with isolated fixed points on such manifolds. We recall from [LR85]:

Definition 4.1. A simply-connected CW-complex $X$ is called an $F C 2$-space, if $X_{(0)}$ is formal and has cup-length at most 2, i.e., $\tilde{H}^{*}(X ; \mathbf{Q}) \cup \tilde{H}^{*}(X ; \mathbf{Q}) \cup$ $\tilde{H}^{*}(X ; \mathbf{Q})=0$.

Remark 4.2. (1) [LR85] If $X$ is rationally homotopy equivalent to a sphere or to a connected sum of products of spheres, i.e.,

$$
X \simeq_{\mathrm{Q}} \sum_{j}^{\sharp}\left(S^{i_{j}} \times S^{n-i_{j}}\right), \quad 2 \leq i_{j} \leq n-i_{j},
$$

then $X$ is an $F C 2$-space.

(2) [LR85] If $M^{n}$ is a 1-connected manifold (Poincaré duality space suffices) with $H_{i}(M ; \mathbf{Q})=0$ for $i \leq k$ with $3 k+1 \geq n$, then $M$ is an $F C 2$-space.

(3) If $M^{n}$ is a 1-connected manifold with $H_{i}(M ; \mathbf{Q})=0$ for $i \leq k$ with $3 k+1 \geq n$ and $\operatorname{index}(M)=0$, then $M$ is rationally homotopy equivalent to a sphere or a connected sum of products of spheres as in (1) above.

In the following, we shall concentrate on 1-connected manifolds $X^{n}$ with

$$
\begin{gathered}
n \text { even, } \chi(X)>0, \operatorname{index}(X)=0 ; \\
H_{i}(X ; \mathbf{Q})=0 \text { for } i \leq k \text { with } 3 k+1 \geq n .
\end{gathered}
$$

The corresponding case with $\chi(X)=0$, in particular with $n$ odd, is already treated in [LR85].

Some ideas and notations from [Rau92] are relevant in the following: Let $X^{n}$ denote a manifold as in (4.1), on which $T$ acts with $X^{T} \neq \varnothing$. The $\mathbf{Q}[e]$-module $H^{*}\left(X_{T} ; \mathbf{Q}\right)$ contains a $\mathbf{Q}[e]$-torsion submodule $\operatorname{Tor}\left(H^{*}\left(X_{T} ; \mathbf{Q}\right)\right)$. In [Rau92] we introduced and investigated the subspaces $I(X)=p^{*}\left(\operatorname{Tor}\left(H^{*}\left(X_{T} ; \mathbf{Q}\right)\right)\right) \subseteq$ $R(X)=p^{*} H^{*}\left(X_{T} ; \mathbf{Q}\right) \subseteq H^{*}(X ; \mathbf{Q})$. An analysis of the derivation $t: H^{*}(X) \rightarrow$ $H^{*-1}\left(X_{T}\right)$ in the Gysin sequence as in [LR85, Rau92] yields a Q-linear map $\tau$ : $H^{*}(X) / R(X) \rightarrow I(X)[e]$ that, combined with evaluation $e v_{1}$ at $e=1$, yields an isomorphism $e v_{1} \circ \tau: H^{*}(X) / R(X) \rightarrow I(X)$ of Q-vector spaces (of odd negativ degree). Similar to [LR85, Chapter 2], this gives rise to an isomorphism of $\mathbf{Q}[e]$-vector spaces

$$
\operatorname{Tor}\left(H^{*}\left(X_{T}\right)\right) \cong I(X)[e] / e \cdot \tau\left(H^{*}(X) / R(X)\right),
$$

and hence,

$$
H^{*}\left(X_{T}\right) \cong I(X)[e] / e \cdot \tau\left(H^{*}(X) / R(X)\right) \oplus(R(X) / I(X))[e],
$$


where we consider $I(X)$ and $R(X) / I(X)$ as Q-vector spaces generating $H^{*}\left(X_{T}\right)$ as a $\mathrm{Q}[e]$-module. We can also describe the trivial part of the cupproduct structure on $H^{*}\left(X_{T}\right)$ :

Lemma 4.3. $\operatorname{Tor}\left(H^{*}\left(X_{T}\right)\right) \cup \tilde{H}^{*}\left(X_{T}\right)=\{0\} \subseteq H^{*}\left(X_{T}\right)$ for a $T$-manifold $X$ satisfying 4.1 .

Proof. All elements in $I(X) \cdot \tilde{H}^{*}\left(X_{T}\right)$ are $\mathrm{Q}[e]$-torsion in dimensions $\geq \frac{2 n+4}{3}$; since $\tau$ is trivial in this range of dimensions, such a torsion element has to be trivial on the nose.

Now, we proceed to construct the cohomology of a possible Borel space for some $T$-action on $X$, denoted as $H_{[e]}^{*}(X):$ If $X$ is a rational homology sphere, we define

$$
H_{[e]}^{*}(X)=H^{*}\left(S(n / 2 \cdot U \oplus \mathbf{R})_{T}\right) \rightarrow 2 \cdot H^{*}(B T),
$$

where $U$ denotes a 1-dimensional free complex $T$-representation, and the map is induced from the inclusion map of the two fixed points of the action induced on the sphere at the Borel space level.

If $X$ is not a rational homology sphere, (4.2) yields a rational homotopy equivalence

$$
X \simeq_{\mathbf{Q}} N=\sum_{1 \leq j \leq k}^{\sharp}\left(S^{i_{j}} \times S^{n-i_{j}}\right) \sharp \sum_{1 \leq j \leq k}^{\sharp}\left(S^{i_{j}^{\prime}} \times S^{n-i_{j}^{\prime}}\right) \sharp \sum_{1 \leq j \leq l}^{\sharp}\left(S^{i_{j}^{\prime \prime}} \times S^{n-i_{j}^{\prime \prime}}\right),
$$

where $i_{j}<\frac{n}{2}$ and odd, $i_{j}^{\prime}, i_{j}^{\prime \prime} \leq \frac{n}{2}$ and even. In this case, $\chi(X)=2 l+2$.

We introduce an auxiliary space $S=\sum_{1 \leq j \leq l}^{\sharp}\left(S^{i_{j}^{\prime \prime}} \times S^{n-i_{j}^{\prime \prime}}\right)$, on which $T$ acts semifreely with $2 l+2$ ixolated fixed points as follows: Start with a linear action with two isolated fixed points of the form $S(U \oplus \mathbf{R}), U$ a free $T$-representation, on each of the spheres. This produces actions with four isolated fixed points with the same tangential representations on every component in the connected sum. Taking a connected sum by identifying and eliminating fixed points in pairs yields the desired action with $\chi(S)=2 l+2$ isolated fixed points.

Inclusion of the fixed point set induces a map $i_{S}:(2 l+2) \cdot B T \rightarrow S_{T}$ on the Borel space level, and the corresponding cohomology homomorphism $i_{S}^{*}: H^{*}\left(S_{T}\right) \rightarrow(2 l+2) \cdot H^{*}(B T)$ becomes an isomorphism after inverting $e$ by the Borel localization theorem.

The cohomology fundamental classes of the spheres in $N$ (cf. 4.3) will be denoted $x_{j}, y_{j}, x_{j}^{\prime}, y_{j}^{\prime}, x_{j}^{\prime \prime}$, resp. $y_{j}^{\prime \prime}$. Then $x_{j} \cup x_{j}^{\prime}=x_{j}^{\prime} \cup y_{j}^{\prime}=x_{j}^{\prime \prime} \cup y_{j}^{\prime \prime}=[N]$, the cohomology fundamental class, whereas all other products vanish. Define

$$
I(X)=\left\langle x_{j}, x_{j}^{\prime}\right\rangle_{\mathbf{Q}}, \quad R(X)=I(X) \oplus\left\langle x_{j}^{\prime \prime}, y_{j}^{\prime \prime}\right\rangle_{\mathbf{Q}},
$$

and

$$
\tau: H^{*}(X) / R(X) \rightarrow I(X)[e]
$$

by

$$
\tau\left(\left[y_{j}\right]\right)=x_{j}^{\prime} \cdot e^{\frac{n-i_{j}-i_{j}^{\prime}-1}{2}}, \quad \tau\left(\left[y_{j}^{\prime}\right]\right)=x_{j} \cdot e^{\frac{n-i_{j}^{\prime}-i_{j}-1}{2}} .
$$

We define, in accordance with (4.2) as a $\mathbf{Q}[e]$-module,

$$
H_{[e]}^{*}(X)=I(X)[e] / e \cdot\left(\tau\left(H^{*}(X) / R(X)\right)\right)[e] \oplus\left\langle y_{j}, y_{j}^{\prime}\right\rangle_{\mathbf{Q}}[e] .
$$


Remark that $\left\langle y_{j}, y_{j}^{\prime}\right\rangle_{\mathbf{Q}}[e] \cong H^{*}\left(S_{T}\right)$ as a $\mathbf{Q}[e]$-module; we use this isomorphism together with Lemma 4.3 to provide $H_{[e]}^{*}(X)$ with a graded product structure. There is a projection homomorphism $P: H_{[e]}^{*}(X) \rightarrow H^{*}\left(S_{T}\right)$ with kernel $I(X)[e] /\left(e \cdot \tau\left(H^{*}(X) / R(X)\right)\right)[e]$, which gets isomorphic after inverting $e$. Together with the map $i_{S}^{*}$ above, we have constructed a graded ring homomorphism

$$
i_{S}^{*} \circ P: H_{[e]}^{*}(X) \rightarrow H^{*}\left(S_{T}\right) \rightarrow(2 l+2) \cdot H^{*}(B T),
$$

which gets an isomorphism after inverting $e$.

Finally, to get back from cohomology algebras to deformations of rational homotopy types, we apply

Lemma 4.4. Let $\mathscr{H}_{i}^{*}, 1 \leq i \leq 3$, denote graded 1-connected Q-algebras, $\mathscr{H}_{2}^{*}$ moreover a $\mathbf{Q}[e]$-algebra, $|e|=2$, which fit into an exact sequence

$$
\cdots \rightarrow \mathscr{H}_{2}^{*-2} \stackrel{\cdot e}{\longrightarrow} \mathscr{H}_{2}^{*} \stackrel{P}{\rightarrow} \mathscr{H}_{3}^{*} \stackrel{\tau}{\rightarrow} \mathscr{H}_{2}^{*-1} \stackrel{\cdot e}{\longrightarrow} \mathscr{H}_{2}^{*+1} \rightarrow \cdots,
$$

where $P$ is a ring homomorphism and $\tau$ a derivation. Let furthermore

$$
\psi: \mathscr{H}_{2}^{*} \rightarrow \mathscr{H}_{1}^{*} \otimes \mathbf{Q}[e]
$$

denote a $\mathbf{Q}[e]$-algebra homomorphism preserving $e$, which becomes an isomorphism after inverting $e$.

Then there are (formal) rational spaces $F, X, X_{[e]}$ and maps $X \stackrel{p}{\rightarrow} X_{[e]}$, resp. $F \times B T_{(0)} \stackrel{j_{[e]}}{\longrightarrow} X_{[e]}$, that induce $P$, resp. $\psi$, in cohomology. In particular, $j_{[e]}^{*}: \mathscr{M}\left(X_{[e]}\right) \rightarrow \mathscr{M}\left(F \times B T_{(0)}\right)$ satisfies Assumption $B$.

Proof. Let $\varphi_{i}:\left(\mathscr{A}_{i}, d_{i}\right) \rightarrow \mathscr{H}_{i}^{*}, i=1,2$, denote (formal 1-connected) dga minimal models. $\mathscr{A}_{2}$ contains a (unique) cocycle $e$ of degree 2 representing the element of the same name in $\mathscr{H}_{2}^{*}$. Hence, the cokernel of the map $\cdot e: \mathscr{A}_{2}$ $\rightarrow \mathscr{A}_{2}$ is a (1-connected) dga $\left(\mathscr{A}_{3}, d_{3}\right)$, and $\varphi_{2}$ induces a dga map $\varphi_{3}: \mathscr{A}_{3} \rightarrow$ $\operatorname{coker}\left(\cdot e: \mathscr{H}_{2}{ }^{*} \rightarrow \mathscr{H}_{2}^{*}\right) \stackrel{P}{\leftrightarrow} \mathscr{H}_{3}{ }^{*}$. Induction on the "Gysin" sequences (4.5) and of the quotient map $\mathscr{A}_{2} \rightarrow \mathscr{A}_{3}$, cf. (1.1), shows that $\varphi_{3}$ induces an isomorphism in cohomology.

In fact, the quotient map $\mathscr{A}_{2} \rightarrow \mathscr{A}_{3}$ induces $P$. Realizing the latter by rational spaces and maps [GM81, BG76] yields the map $p: X \rightarrow X_{[e]}$. According to [GM81], the map $\psi$ can be lifted to give a dga map $\Psi: \mathscr{A}_{2} \rightarrow \mathscr{A}_{1} \otimes \mathbf{Q}[e]$. A realization of this map gives us the map $j_{[e]}: F \times B T_{(0)} \rightarrow X_{[e]}$.

The following final result does not yet give us $T$-actions on the manifolds considered here, but should be considered as a first approximation step: Let $X$ be a manifold satisfying (4.1) with $\chi=\chi(X)>0$. Let $U_{i}, 1 \leq i \leq \chi$, denote disjoint open disk neighbourhoods of $\chi$ points in $X$, and define $U=\bigcup_{1}^{\chi} U_{i}$, and $M=X \backslash U$. Its boundary $\partial M$ may be (nonequivariantly) identified with the space $S=\bigcup_{1}^{\chi} S_{i}$, where $S_{i}=S(V), 1 \leq i \leq \chi$, and $V$ denotes a complex free $T$-representation of real dimension $n$.

Proposition 4.5. There is a rational space $\tilde{M}$ rationally homotopy equivalent to $M$ supporting a free $T_{(0)}$-action. Furthermore, there is a $T_{(0)}$-equivariant map $I: S_{(0)} \rightarrow \tilde{M}$ that, up to rational cohomology, corresponds to the inclusion $S=\partial M \subset M$. 
Proof. Lemma 4.4 is applied to the case $\mathscr{H}_{1}^{*}=(2 l+2) \cdot H^{*}(B T), \mathscr{H}_{2}^{*}=$ $H_{[e]}^{*}(X), \mathscr{H}_{3}^{*}=H^{*}(X)$, and $\Psi=i_{S}^{*} \circ P$ from 4.4. As a result, we obtain a map $j_{[e]}: \chi \cdot B T_{(0)} \rightarrow X_{[e]}$, which satisfies Assumption B. We want to apply Propositions 2.8 and 2.11, and have to check Assumptions A through E. The inclusion of $\chi$ points into $X$ obviously satisfies Assumptions A, C, and E. Assumption D is certainly satisfied (cf. Remark 1.9) when $X_{[e]}$ is replaced by $S_{T}$, cf. (4.4). Since the images of $H^{*}\left(X_{[e]}\right)$ and of $H^{*}\left(S_{T}\right)$ in $H^{*}(\chi \cdot B T)$ coincide, Assumption D is satisfied for $X$ as well.

Hence, Propositions 2.8 and 2.11 yield maps

$$
\chi \cdot \mathrm{C} P_{(0)}^{\frac{n}{2}-1} \rightarrow M_{[e]} \rightarrow B T_{(0)} .
$$

The pullback along these maps of the classifying fibre bundle $E T_{(0)} \downarrow B T_{(0)}$ yields the required equivariant map of free $T_{(0)}$-spaces $S_{(0)} \rightarrow \tilde{M} \simeq_{\mathrm{Q}} M$.

Remark 4.6. For manifolds satisfying (4.1), but with negative Euler characteristic, one can perform a similar construction with $F=\sum_{j}^{\sharp}\left(S^{3} \times S^{3}\right)$. Modulo fundamental group problems, one might also choose $F$ to be an orientable surface.

\section{REFERENCES}

[All78] C. Allday, On the rational homotopy of the fixed point set of torus actions, Topology 17 (1978), 95-100.

[AP93] C. Allday and V. Puppe, Cohomological methods in transformation groups, Cambridge Stud. Adv. Math., vol. 32, Cambridge Univ. Press, London and New York, 1993.

[BG76] A. K. Bousfield and V. K. A. M. Gugenheim, On PL de Rham theory and rational homotopy type, Mem. Amer. Math. Soc. 179 (1976).

[Ger64] M. Gerstenhaber, On the deformation of rings and algebras, Ann. of Math. (2) 79 (1964), 59-103.

[GM81] P. A. Griffiths and J. W. Morgan, Rational homotopy theory and differential forms, Progr. Math., vol. 16, Birkhäuser, Basel and Boston, 1981.

[Hal83] S. Halperin, Lectures on minimal models, Mém. Soc. Math. France, vol. 9/10, Soc. Math. France, 1983.

[LR85] P. Löffler and M. Raußen, Symmetrien von Mannigfaltigkeiten und rationale Homotopietheorie, Math. Ann. 272 (1985), 549-576.

[Mil79] T. J. Miller, On the formality of $(k-1)$ connected manifolds of dimension less than or equal to $4 k-2$, Illinois J. Math. 23 (1979), 253-258.

[Rau92] M. Raußen, Rational cohomology and homotopy of spaces with circle action, Algebraic Topology-Homotopy and Group Cohomology, Proc., Barcelona 1990 (J. Aguadé, M. Castellet, and F. R. Cohen, eds.), Lecture Notes in Math., vol. 1509, Springer-Verlag, Berlin and New York, 1992, pp. 313-325.

[Rau94] _Symmetries on manifolds via rational homotopy theory, in preparation.

[Sul77] D. Sullivan, Infinitesimal computations in topology, Inst. Hautes Etudes Sci. Publ. Math. 47 (1977), 269-331.

Institute for Electronic Systems, Aalborg University, Department of Mathematics and Computer Science, Fredrik Bajers Vej 7E, DK - 9220 Aalborg $\varnothing$, Denmark

E-mail address: raussen@iesd. auc.dk 